\title{
Méthode simple pour le dosage du magnésium dans le lait, utilisant la spectrophotométrie par absorption atomique (S.A.A.)
}

\author{
par \\ Razi RAZIFARD D.V.M. M.Sc. \\ Chaire de la Nutrition Animale \\ Faculté vétérinaire de Téhéran (Iran)
}

\section{INTRODUCTION}

Le dosage de certaines substances chimiques par la méthode S.A.A. a été pour la première fois mis en œuvre en 1955 par Walsh. En 1959 et 1960, David a pu montrer que, lors du dosage du $\mathrm{Mg}$ par S.A.A., il y avait interférence des silicates, des phosphates, des sulfates, de l'aluminium, etc., interférence que supprime la présence d'ions calcium. Depuis, cette méthode a été longtemps répandue, notamment en ce qui concerne l'évaluation des taux de $\mathrm{Mg}$ de très divers produits :

Sérum sanguin (Willis 1960 et Hurst, Swing 1963), urine (Willis 1961), différentes substances alimentaires (Heckman 1967), lait après dessiccation (Murthy et Rhea 1967). Par ailleurs, cette méthode utilisant la S.A.A. s'est révélée être plus précise et plus sensible que les autres méthodes chimiques utilisées de façon plus courante (Roach et al. 1968) et (Lee et Campbell 1969).

Dans le présent travail, nous avons appliqué cette méthode S.A.A. à l'évaluation des taux du $\mathrm{Mg}$ dans des laits traités soit par " digestion ", soit par " dilution » et nous avons étudié les résultats du dosage en fonction du type de préparation.

\section{Matériel}

\section{MATERIEL ET METHODES}

- spectrophotomètre par absorption atomique,

- acide nitrique $\mathrm{d}=1,42$ (pur),

- acide perchlorique $\mathrm{d}=1,70$ (pur), 
- solution de chlorure de lanthane $\left(\mathrm{LaCl}_{3}, 7 \mathrm{H}_{2} \mathrm{O}, 174 \mathrm{~g}, \mathrm{NO}_{3} \mathrm{H}\right.$ $200 \mathrm{ml}, \mathrm{H}_{2} \mathrm{O}$ distillée, q.s.p. $1000 \mathrm{ml}$ ).

\section{Prélèvements}

On disposait de 42 échantillons de lait provenant de six vaches.

Préparation des échantillons :

\section{1) Par dilution}

- Après écrémage par centrifugation $(3500 \mathrm{t} / \mathrm{mn}$ ) on dilue $2 \mathrm{ml}$ du lait dans $23 \mathrm{ml}$ d'eau distillée.

- On ajoute à $5 \mathrm{ml}$ de la solution obtenue $2,5 \mathrm{ml}$ de chlorure de lanthane et on complète à $25 \mathrm{ml}$ avec de l'eau distillée.

2) Par digestion (selon la méthode proposée par Smith en 1969)

- On ajoute à $2 \mathrm{ml}$ du même lait $4 \mathrm{ml}$ d'acide nitrique et $4 \mathrm{ml}$ d'acide perchlorique.

- On porte le mélange au four Kdjeldal pendant $30 \mathrm{mn}$ et on laisse refroidir jusqu'à température ambiante.

- On complète à $25 \mathrm{ml}$ avec de l'eau distillée.

- On ajoute à $5 \mathrm{ml}$ de la solution obtenue, $2,5 \mathrm{ml}$ de chlorure de lanthane et on complète à $25 \mathrm{ml}$ avec de l'eau distillée.

\section{RESULTATS ET DISCUSSION}

Les taux de $\mathrm{Mg}$ obtenus sur le lait préparé par " digestion " fluctuent entre 10 et $15,66 \mathrm{mg}$ p. 100 (moyenne $\mathrm{X}^{-}=12,34 \mathrm{mg} \mathrm{p} .100$ ).

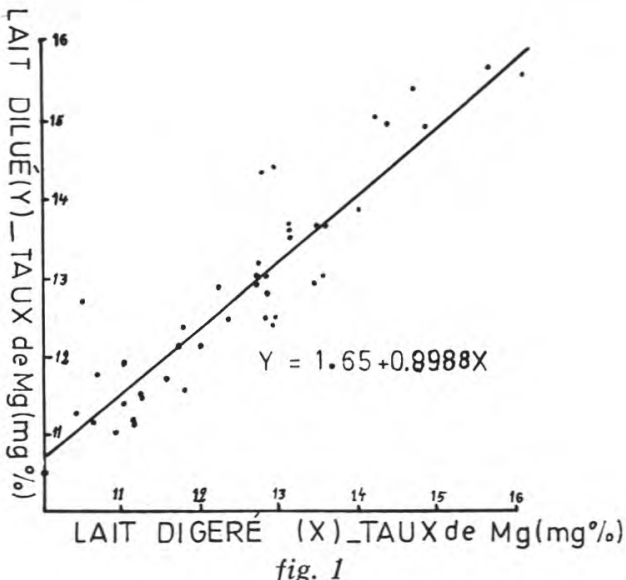

Taux de magnésium dans le lait digéré et dans le lait dilué 
Les taux de Mg obtenus sur le lait préparé par « dilution » fluctuent entre 10,50 et $15,45 \mathrm{mg}$ p. 100 (moyenne $\mathrm{Y}^{-}=12,74 \mathrm{mg} \mathrm{p} .100$ ). Les résultats se trouvent être très proches du chiffre de $12,60 \mathrm{mg}$ p. 100 de l'Agricultural Research Council (1965).

Il y a une corrélation $(\mathrm{P}<0,001)$ entre les résultats obtenus par les deux méthodes. Le coefficient de corrélation obtenue est 0,9232 et la fraction de régression $\mathrm{Y}$ sur $\mathrm{X}$ est $\mathrm{Y}=1,65+0,8988 \mathrm{X}$ (fig. 1). La comparaison des moyennes, effectuée par l'étude de l'ensemble des variances, ne montre aucune différence significative entre les résultats obtenus.

En conclusion, le mode de préparation des échantillons (dilution ou digestion) ne semble pas influer sur l'évaluation des taux de $\mathbf{M g}$ par la méthode S.A.A. dans le lait. La préparation par dilution des échantillons présente l'avantage d'être plus facilement réalisable.

\section{Ré s u mé}

L'évaluation des taux de $\mathrm{Mg}$ dans le lait a été réalisée par spectrophotométrie par absorption atomique après traitement des échantillons par digestion et par dilution. Les taux moyens obtenus sont respectivement $\mathrm{X}^{-}=12,34$ et $\mathrm{Y}^{-}=12,74 \mathrm{mg} \mathrm{Mg} \mathrm{p} .100$. Une corrélation étroite $(\mathrm{P}<0,001)$ a été mise en évidence entre les résultats obtenus par ces deux méthodes. Le mode de préparation des échantillons semble ne pas influer sur l'évaluation du $\mathbf{M g}$ dans le lait par S.A.A., mais la méthode de préparation par dilution présente l'avantage d'être plus facilement réalisable.

\section{S u m m a r y}

For magnesium determination in milk 2 techniques, based on atomic absorption spectrophotometry, were compared from the point of view of simplicity and accuracy.

Mean value of magnesium in wet digested milk samples was $\mathrm{X}^{-}=12.34 \mathrm{mg} \mathrm{p} .100$ and in the same samples when diluted was $\mathrm{Y}^{-}=12,74 \mathrm{mg}$ p. 100. The magnesium values of wet digested and diluted milk were highly correlated $(\mathrm{P}<0.001)$. The determination of magnesium in milk by the dilution method proved to be accurate and less laborious than the method involving digestion of milk.

\section{Références bibliographiques}

DAvid (D. J.) (1959). - Determination of calcium in plant material by atomic absorption spectrophotometry. Analyst. Lond., 84, 536. 
David (D. J.) (1960). - The application of atomic absorption to chemical analysis. Analyst, Lond., 85, 779.

Heckman (M.) (1967). - Minerals in feeds by atomic absorption spectrophotometry. J. Ass. offic. Anal. Chem., 50, 45.

Hurst (R. J.) and Mc Swiney (R. R.) (1963). - Hilger atomic absorption apparatus used to determine magnesium in blood plasma. Hilger J., 8, 25.

LeE (J.) and CAMPBell (M.) (1969). - Atomic absorption spectrophotometric and ethylenediaminetetraacetate titration methods for calcium and magnesium. J. Dairy. Sci., 52, 121.

Murthy (G. K.) and RheA (U.) (1967). - Determination of major cations in milk by atomic absorption spectrophotometry. J. Dairy Sci., 50, 313.

Roach (A. G.) Sanderson (P.) and Williams (D. R.) (1968). - Determination of trace amounts of copper, zinc and magnesium in Animal Feeds by atomic absorption spectrophotometry. Analyst, Lond., 93, 42.

Sмiтн (R. H.) (1969). - Communication personnelle.

WALSH (A.) (1955). - The application of atomic absorption spectra to chemical analysis. Spectrochim. Acta, 7, 108.

Willis (J. B.) (1960). - Determination of metals in blood serum by atomic absorption spectroscopy. I. Calcium. II. Magnesium. Spectrochim. Acta., $16,259$.

Willis (J. B.) (1961). - Determination of calcium and magnesium in urine by atomic absorption spectroscopy. Analyt. Chem., 33, 556. 